\title{
The effect of exercise training on adiponectin receptor expression in KKAy obese/diabetic mice
}

\author{
Hu Huang*, Kaoruko Tada lida*, Hirohito Sone, \\ Tomotaka Yokoo ${ }^{1}$, Nobuhiro Yamada ${ }^{1}$ and Ryuichi Ajisaka \\ Doctoral Program of Sports Medicine, Graduate School of Comprehensive Human Sciences, University of Tsukuba, 1-1-1 Tennodai, Tsukuba-shi, Ibaraki \\ 305-8575, Japan \\ ${ }^{1}$ Doctoral Program in Advanced Biomedical Applications, Graduate School of Comprehensive Human Sciences, University of Tsukuba, 1-1-1 Tennodai, \\ Tsukuba-shi, Ibaraki 305-8575, Japan \\ (Requests for offprints should be addressed to K T lida; Email: IIDAK@taiiku.tsukuba.ac.jp) \\ *( $\mathrm{H}$ Huang and K T lida contributed equally to this paper $)$
}

\begin{abstract}
Adiponectin is an adipocyte-derived factor that plays a pivotal role in lipid and glucose metabolism. Recently, two types of adiponectin receptors (AdipoR1 and AdipoR2) were identified. We investigated whether exercise training (ET) or dietary restriction (DR) affects the expression of adiponectin receptors in skeletal muscle and liver, thereby improving glucose and lipid metabolism in KKAy mice. KKAy mice were subjected to 8 weeks of exercise training or food restriction. Following the experimental protocol, an intravenous glucose tolerance test and an intraperitoneal insulin tolerance test were performed in addition to the measurement of blood lipid and adiponectin concentrations. The mRNA levels of adiponectin, adiponectin receptors and genes that are putatively regulated by the adiponectin receptors were also analyzed. Both the 8-week exercise training and food restriction protocol improved insulin resistance in KKAy mice but did not alter plasma adiponectin concentration nor its mRNA expression. In comparison with C57BL/6 mice,
\end{abstract}

AdipoR1 expression level was significantly decreased in skeletal muscle and AdipoR2 expression level was significantly increased in the liver in KKAy mice. After the 8-week experimental protocol, the expression level of AdipoR1 mRNA was approximately $1 \cdot 8$-fold greater in the skeletal muscle and 1.3-fold greater in the liver, and the level of AdipoR2 mRNA was 30\% less in the liver of the ET group as compared with the control group. Additionally, in the ET group, mRNA expression of acyl coenzyme A-oxidase and carnitine palmitoyl transferase 1 (CPT1) was greater in the liver but not in skeletal muscle. In contrast, no significant changes were observed in the expression of genes encoding the adiponectin receptors in addition to other genes except for CPT1 in the DR group. These findings suggest that chronic exercise training affects the expression level of adiponectin receptors thereby improving insulin resistance in KKAy mice.

Journal of Endocrinology (2006) 189, 643-653

\section{Introduction}

It is well known that adipose tissue secretes multiple proteins that mediate various biological functions. These proteins are known as adipocytokines, which include tumor necrosis factor- $\alpha$, leptin, plasminogen activator inhibitor type-1 (PAI-1) and resistin (Hotamisligil et al. 1993, Lonnqvist et al. 1995, Shimomura et al. 1996, Steppan et al. 2001). Several years ago, a new adipocytokine, referred to as adiponectin, was identified. Adiponectin was first identified as a $30 \mathrm{kDa}$ cytokine (also reported as apM-1, AdipoQ and Acrp30) (Scherer et al. 1995, Hu et al. 1996, Maeda et al. 1996), and its mRNA and plasma levels were decreased in obesity and diabetes (Arita et al. 1999, Hu et al. 1996). In addition, various recent studies have shown that adiponectin influences glucose homeostasis and insulin sensitivity. Increased serum adiponectin concentration is associated with improvement in glucose tolerance and insulin sensitivity in both monkey and human (Hotta et al. 2001, Weyer et al. 2001, Yang et al. 2001). Yamauchi et al. (2001) have shown that it is possible to improve insulin resistance in obese mice by administration of recombinant adiponectin. These findings suggest that adiponectin plays a key role in the pathogenesis of insulin resistance and diabetes.

Exercise training is a useful therapy for improving insulin sensitivity. Although less is known about the association of adiponectin with the beneficial effects of exercise, several recent studies have examined whether exercise training affects plasma adiponectin concentrations. 
However, most of these studies indicate no significant changes in plasma adiponectin concentration after exercise training in spite of the variation in the subject's characteristics (healthy or diabetes), training protocols (single bout, intermittent or endurance) and intensities of the exercise (Hulver et al. 2002, Boudou et al. 2003, Kraemer et al. 2003).

Recently, receptors for adiponectin (AdipoR) have been cloned (Yamauchi et al. 2003). There are two subtypes of AdipoR, adiponectin receptor 1 (AdipoR1) and adiponectin receptor 2 (AdipoR2); AdipoR1 is ubiquitously expressed with the greatest expression found in skeletal muscle, whereas AdipoR2 is mainly expressed in the liver. In addition, the finding that the inhibition of AdipoR expression results in the suppression of glucose uptake in vitro (Yamauchi et al. 2003) suggests that the proposed effect of adiponectin on glucose homeostasis in individual organs might be, at least in part, regulated at the expression level of the adiponectin receptor.

Skeletal muscle and liver are major organs involved in glucose metabolism. In addition, within skeletal muscle the expression and activation of some molecules involved in glucose metabolism, such as the glucose transporter protein GLUT-4 and 5-AMP-activated protein kinase, are known to be modified by exercise training (Friedman et al. 1990, Kern et al. 1992, Winder \& Hardie 1996). Therefore, the aim of the present study was to clarify whether exercise training regulates the expression levels of AdipoR in skeletal muscle and liver, thereby affecting insulin sensitivity and the diabetic state. We addressed this aim by examining the effect of exercise training on AdipoR expression in male KKAy mice. In this study, the effect of food restriction on AdipoR expression was also assessed to determine whether exercise training affects the expression of AdipoR exclusively.

\section{Materials and Methods}

\section{Experimental animals and protocols}

Eight-week-old KKAy male mice $(n=18)$ from Clea Japan (Tokyo, Japan) were housed under controlled temperature and lighting conditions of $20^{\circ} \mathrm{C}-22{ }^{\circ} \mathrm{C}, 12-\mathrm{h}$ light-darkness cycle; a regular diet containing $27 \%$ protein, $13 \%$ lipid, and $60 \%$ carbohydrate (Oriental Yeast Inc, Tokyo, Japan) was available ad libitum until the start of the experiment. Once the experimental protocol was initiated, KKAy mice were divided into 3 groups: exercise training $(\mathrm{ET} ; n=6)$, dietary restriction $(\mathrm{DR} ; n=6)$ and control (CON; $n=6)$. Body weight and food consumption of all mice were measured daily. Age-matched C57BL/6 $(n=6)$ male mice (Clea Japan) were also prepared as the wild-type control for comparison of mRNA expression.

Following acclimatization to running on a treadmill (Muromachi, Tokyo, Japan), mice in the ET group were exercised at $15 \mathrm{~m} / \mathrm{min}$ for $45 \mathrm{~min}$ per day in the early evening, 5 days per week for a total of 8 weeks. We measured the daily food consumption of the animals in the control group, and mice in the DR group were provided with $70 \%$ of the volume of food that control mice consumed. Following a 12-h fasting period at the end of the study, the mice were killed and the biceps femoris muscle, liver and epididymal fat tissue were removed and immediately frozen in liquid nitrogen. A part of the epididymal fat tissues was fixed in 10\% formalin solution for morphological analysis (see below). All protocols were approved by the institutional Animal Care and Use Committee of the University of Tsukuba, Japan.

\section{Blood chemistry assay}

After an 8-week period, blood samples of KKAy mice were collected from the orbita of anesthetized animals following a 12- to 14-h overnight fasting period. Total cholesterol, triglyceride, free fatty acid (FFA) and glucose concentrations were assayed spectrophotometrically using a commercially available kit (Wako Pure Chemical Industries, Osaka, Japan). Plasma insulin and adiponectin concentrations were assessed using a mouse Lebis insulin ELISA kit (Shibayagi, Gunma, Japan) and a mouse adiponectin ELISA kit respectively (Otsuka, Tokyo, Japan). The index of homeostasis model assessment of insulin resistance (HOMA-R: insulin $(\mathrm{mU} / \mathrm{l}) \times$ glucose $(\mathrm{mM}) /$ 22.5) (Matthews et al. 1985) of each animal was calculated from fasting plasma glucose and insulin concentrations.

\section{Intravenous glucose tolerance test (IVGTT) and intraperitoneal} insulin tolerance test (IPITT)

One week prior to the last day of the experiment, an IVGTT and IPITT were performed. After an overnight fast, IVGTT was performed by intravenous injection of a $20 \%$ glucose solution $(2 \mathrm{~g} / \mathrm{kg})$ via the tail vain. Blood samples were collected before and 5, 30, 60, and $120 \mathrm{~min}$ after the injection. For IPITT, after a $4-\mathrm{h}$ fast, an intraperitoneal injection of $2 \mathrm{U} / \mathrm{kg}$ human rapid insulin (Eli Lilly Co, Indianapolis, IN, USA) was given. Blood samples were collected before and 15, 30, 60, 90, and $120 \mathrm{~min}$ after the injection. Blood glucose levels were measured with a glucose C test kit (Wako Pure Chemical Industries). For the IPITT, the response of blood glucose levels was expressed as a percentage of the values before insulin injection.

\section{Morphological analysis of the white adipose tissue}

Serial sections were taken from the post-fixed epididymal fat tissues removed from the killed mice, then stained with Massons' trichrome stain. Stained sections were photographed digitally using an optical microscope and a digital camera (Coolpix; Nikon, Tokyo, Japan), and the images 
Table 1 Body weight and blood chemistry analyses following an overnight fasting period at the end of the study

\begin{tabular}{|c|c|c|c|}
\hline & $\mathrm{CON}$ & DR & ET \\
\hline Body weight (g) & $42 \cdot 1 \pm 1 \cdot 5$ & $38 \cdot 6 \pm 2 \cdot 9^{*}$ & $42 \cdot 9 \pm 1 \cdot 9$ \\
\hline FPG (mg/dl) & $139 \pm 47$ & $117 \pm 34^{*}$ & $67 \pm 20^{*}$ \\
\hline Insulin $(\mathrm{mU} / \mathrm{l})$ & $41 \pm 15$ & $11 \pm 3^{*}$ & $24 \pm 11^{*}$ \\
\hline HOMA-R & $12 \cdot 11 \pm 4 \cdot 4$ & $3 \cdot 86 \pm 1 \cdot 9^{*}$ & $3 \cdot 84 \pm 1 \cdot 3^{*}$ \\
\hline Total cholesterol (mg/dl) & $88 \cdot 1 \pm 25 \cdot 3$ & $50 \cdot 4 \pm 11 \cdot 5^{*}$ & $63 \cdot 9 \pm 23 \cdot 3$ \\
\hline Triglyceride $(\mathrm{mg} / \mathrm{dl})$ & $99 \cdot 0 \pm 44 \cdot 7$ & $44 \cdot 0 \pm 23 \cdot 5^{*}$ & $80 \cdot 0 \pm 30 \cdot 2$ \\
\hline Free fatty acid (mEq/l) & $0 \cdot 75 \pm 0 \cdot 15$ & $0 \cdot 5 \pm 0 \cdot 12^{*}$ & $0 \cdot 5 \pm 0 \cdot 11^{*}$ \\
\hline
\end{tabular}

Values are means \pm S.D. HOMA-R (index of homeostatis model assessment of insulin resistance) $=$ fasting plasma glucose $(\mathrm{mM}) \times$ fasting insulin $(\mathrm{mU} / \mathrm{l}) / 22 \cdot 5 .{ }^{*} P<0 \cdot 05$ compared with the control group. FPG, fasting plasma glucose; $\mathrm{CON}$, control group; DR, dietary restriction group; $\mathrm{ET}$, exercise training group.

were transferred to computer medium. The size of 100 cells picked randomly from each sample was calculated as a pixel number, and then converted into the area $\left(\mu \mathrm{m}^{2}\right)$ using computer software (Adobe Systems, San Jose, CA, USA).

\section{Real time PCR}

Total RNA was extracted from the epididymal fat tissues with TRIzol (Invitrogen), and $1 \mu \mathrm{g}$ RNA was reversetranscribed into complementary DNA (cDNA) using a Takara RNA PCR kit (Takara, Tokyo, Japan) according to the manufacturer's instructions. A total of $2 \mu \mathrm{cDNA}$ was used and amplification was performed in duplicate in an ABI PRISM 7900 Sequence Detector (PE Applied Biosystems, Foster City, CA, USA) using the following primer sets: forward primer AAG GAC AAG GCC GTT CTC T, reverse primer CGC ACG ATT TCC CTC TCA GCT G for mouse adiponectin, and forward primer CA GGG TGT GAG TTG GGG AAT G, reverse primer CGC ACG ATT TCC CTC TCA GCT G for mouse $\beta$-actin. The relative amount of each adiponectin transcript was normalized to the amount of $\beta$-actin transcript in the same cDNA.

\section{Northern blot analysis}

Total RNA was isolated with Trizol, and then $10 \mu \mathrm{g}$ RNA were subjected to electrophoresis in a $1 \%$ formaldehyde agarose gel and transferred to a nylon membrane (Hybond N; Amersham). Using the reverse transcriptionpolymerase chain reaction (RT-PCR) method, cDNA for mouse AdipoR1 (470-602) and full-length peroxisome proliferator-activated receptor- $\delta$ (PPAR $\delta$ ) were synthesized from muscle RNA, and AdipoR2 (720-1185) and full-length peroxisome proliferator-activated receptor- $\alpha$ $(\operatorname{PPAR} \alpha)$ were synthesized from liver RNA. The cDNA for mouse acyl-CoA oxidase (ACO), carnitine palmitoyltransferase 1 (CPT1) and PPAR $\alpha$ were a kind gift from Dr Matsuzaka (University of Tsukuba, Japan). The fragments were analyzed by the dideoxy chain termination method and labeled to generate the ${ }^{32} \mathrm{P}$-labeled probes for Northern blot hybridization. The filters were hybridized with labeled probe for $2 \mathrm{~h}$ at $65^{\circ} \mathrm{C}$, and then washed three times in $0.1 \times \mathrm{SSC}, 0.1 \times \mathrm{SDS}$ for $20 \mathrm{~min}$ at $65{ }^{\circ} \mathrm{C}$. The autoradiographs were quantified by densitometry with the BAS2500 Bioimaging Analyzer (Fuji, Tokyo, Japan). Each quantification result was normalized to the signal generated from acidic ribosomal protein 36B4 mRNA in the same sample.

\section{Statistical analysis}

Data were expressed as means \pm S.D. Individual variables were compared using ANOVA, and repeated measures ANOVA was used for variables collected repeatedly from the same animal in an experiment. The statistical software package STATVIEW (SAS Institute Inc, Cary, NC, USA) was used in all analyses. $P$ values $<0.05$ were considered to be statistically significant.

\section{Results}

\section{Blood chemistry parameters}

Before the experiment, we measured the baseline fasting plasma glucose level (FPG) of each animal, and confirmed that there were no significant differences in FPG among the groups. Blood chemistry parameters after the 8-week experimental protocol are shown in Table 1. Although the mice in the control group at 16 weeks of age exhibited hyperglycemia as compared with the 8 weeks of age time point, the FPG level in the ET group at 16 weeks of age was not elevated compared with the 8 -week time point and was significantly lower than in the other groups at the same time point. The fasting insulin levels in both the DR and ET groups were significantly lower than in the control group. The HOMA-R levels in both the ET and DR groups were significantly lower than in the control 
group, suggesting that both exercise training and dietary restriction improved insulin sensitivity in KKAy mice.

To evaluate the effect on lipid metabolism, we also measured the levels of plasma total cholesterol, triglyceride and FFA. In the DR group, fasting total cholesterol and triglyceride levels were significantly lower as compared with those in the control group. In contrast, total cholesterol and triglyceride levels in the ET group did not significantly differ from those in the control group. The FFA levels were significantly lower in both the DR and ET groups.

\section{Glucose and insulin tolerance tests}

To determine whether exercise training and dietary restriction have a beneficial effect on insulin sensitivity, we performed an IVGTT and an IPITT at 7 weeks of the study. As shown in Fig. 1A, blood glucose levels in both the ET and DR groups were significantly lower than those in the control group. Blood glucose levels were significantly lower at 30, 60, and $120 \mathrm{~min}$ in the ET group, and at 60 and $120 \mathrm{~min}$ in the DR group following glucose load as compared with time-matched samples of the control group.

For the IPITT (Fig. 1B), $15 \mathrm{~min}$ after insulin load, blood glucose levels in the ET group were about $20 \%$ lower than those in the control group $(P<0 \cdot 05)$, whereas no significant changes in glucose levels were observed at any of the time points between the DR and control groups.

Body weight, adipose tissue cell size and adiponectin expression levels

Prior to the initiation of the experimental protocol, there were no significant differences in body weight among the groups. Following the 8-week experimental intervention, the body weight of the DR group was significantly lower compared with both the ET and control groups. There was no significant difference between the ET group and the control group (Table 1).

Since a relationship has been reported between the serum levels of some adipocytokines and adipose cell size, we also assessed white adipose tissue cell size in each animal. As shown in Fig. 2, white adipose tissue cell size was significantly smaller in both the ET and the DR groups compared with the control group, although they were not significantly different from each other. To assess the effect of exercise training and dietary restriction on adiponectin secretion, we also measured the plasma adiponectin concentration. Following the 8-week study period, plasma adiponectin concentration levels were not affected by exercise training or diet restriction. Similarly, among groups no significant difference was observed in the levels of mRNA expression analyzed by real-time PCR on white adipose tissues (data not shown).

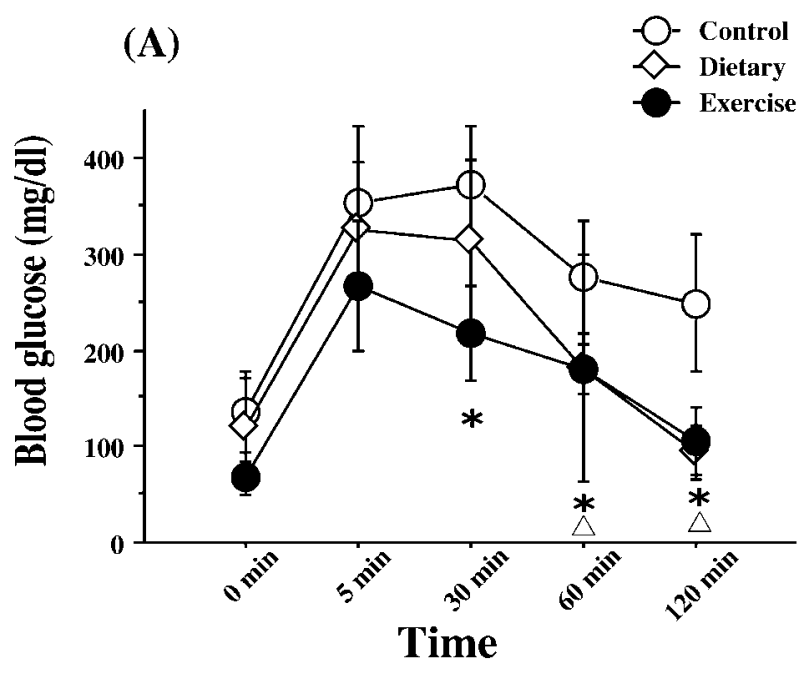

(B)

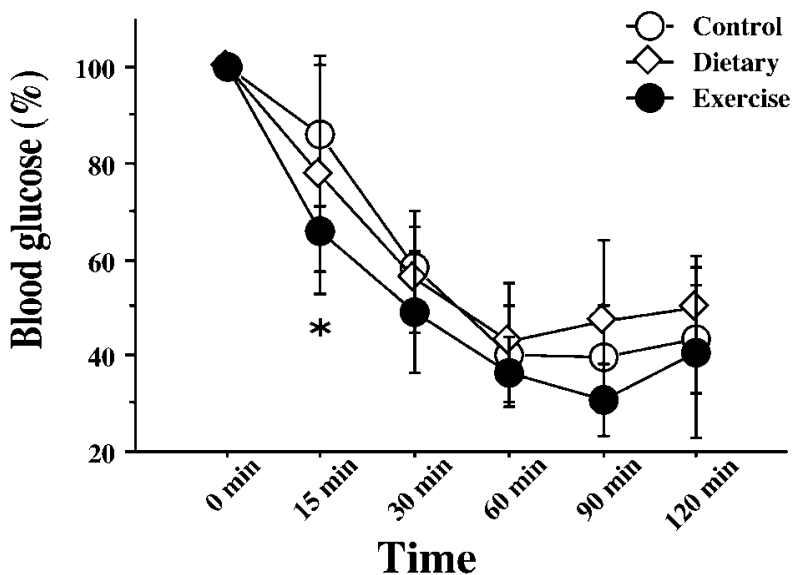

Figure 1 (A) Intravenous glucose tolerance test. The time course of plasma glucose concentration after intravenous glucose injection is shown. Results are expressed as means \pm S.D. (B) Intraperitoneal insulin tolerance test. The time course of plasma glucose concentration after intraperitoneal insulin loading is shown. Results are calculated as a percentage of the initial value in each mouse and expressed as the mean \pm S.D. ${ }^{*} P<0 \cdot 05$, control vs exercise training group; $\triangle P<0 \cdot 05$, control vs dietary restriction group.

Adiponectin receptor $m R N A$ expression in skeletal muscle, liver and white adipose tissue

Northern blot analysis was performed on the RNA obtained from both skeletal muscle and liver, primary organs involved in the expression of the adiponectin receptor. In comparison with age-matched C57BL/6 lean mice, KKAy mice in the control group exhibited lower expression levels of AdipoR1 mRNA in skeletal muscles and higher expression levels of AdipoR 2 mRNA in the liver (Fig. 3). As shown in Fig. 4, exercise training resulted in a significant increase in the mRNA expression of AdipoR1 (but not of AdipoR2) in skeletal muscle of 

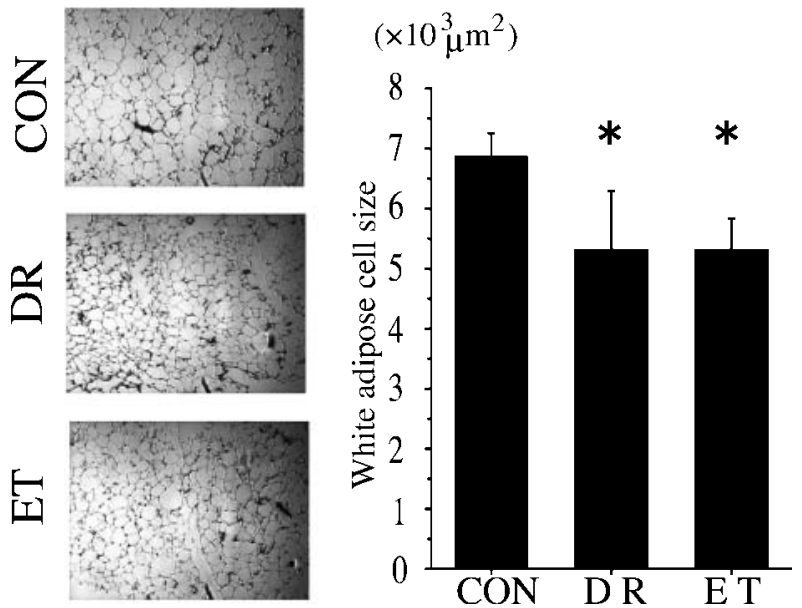

Figure 2 The size of adipose cells in epididymal fat tissue. Epididymal fat tissues removed from mice were fixed and stained with Massons' trichrome stain (left panels). One hundred adipose cells were randomly picked from each sample. The size of each cell was calculated and the average size was measured (right panel). Each bar represents the mean \pm S.D. ${ }^{*} P<0.05$ compared with the control group. CON, control group; DR, dietary restriction group; $\mathrm{ET}$, exercise training group.

KKAy mice. The level of AdipoR1 mRNA in the ET group was about $1 \cdot 8$-fold greater than that in the control group $(P<0 \cdot 05)$. In contrast, in the DR group, no significant changes were observed in either AdipoR1 or AdopoR 2 mRNA expression in the skeletal muscle (Fig. 4 upper panels). In the liver (Fig. 4 middle panels), the level of AdipoR1 mRNA expression in the ET group was significantly (about 1.3-fold) greater than that in the control group, whereas liver AdipoR 2 mRNA expression in the ET group was significantly lower than that in the control group $(P<0 \cdot 05)$. Similar to the results found in skeletal muscle, the levels of both AdipoR 1 and AdopoR2 mRNA expression in the liver of the DR group were not significantly different from those in the control group.

Additionally, we examined the mRNA levels of adiponectin receptors in white adipose tissue. Although the expression levels of both AdipoR1 and AdipoR2 in white adipose tissue of KKAy mice were significantly lower compared with those of C57BL/6 mice (Fig. 3), neither exercise training nor diet restriction affected them (Fig. 4 lower panels).

$m R N A$ expression of PPARa, ACO, CPT1, and PPAR $\delta$ in skeletal muscle and liver

In skeletal muscle and liver, we quantified mRNA levels of ACO and CPT1 (key enzymes involved in fatty-acid oxidation) and of PPAR $\alpha$. In skeletal muscle, we also examined the level of PPAR $\delta$ mRNA expression. As shown in Fig. 5A, in skeletal muscle there was no difference in mRNA expression levels of ACO, CPT1,
$\operatorname{PPAR} \alpha$, and $\operatorname{PPAR} \delta$ in the $\mathrm{ET}$ or the $\mathrm{DR}$ groups compared with the control group, whereas in the liver ACO and CPT1 mRNA expression levels were significantly increased in the ET group $(P<0.05$ for both parameters) (Fig. 5B). Also, in the DR group CPT1 mRNA expression levels were significantly greater than those of the control group in the liver $(P<0 \cdot 05)$, but ACO mRNA expression levels were not significantly different (Fig. 5B).

\section{Discussion}

To the best of our knowledge, this is the first study to assess the effects of exercise training on adiponectin receptor expression in an animal model of spontaneous diabetes. KKAy mice used in this study exhibit moderate hyperglycemia and obesity and have elevated plasma insulin levels; therefore, this animal model has been established as an available model of type 2 diabetes possessing insulin resistance. Previously, there have been no reports that describe the effect of sustained exercise training on the diabetic state or insulin sensitivity in KKAy mice. We demonstrated that both an eight-week exercise training and an eight-week dietary restriction protocol reduced the HOMA-R levels. We also demonstrated a significant improvement in the diabetic state assessed by IVGTT in both the ET and DR groups. In addition, a significant reduction in plasma glucose level by insulin loading was observed only in the ET group. These results indicate that chronic exercise training plays a beneficial role in the improvement of insulin resistance in KKAy mice.

On the other hand, although food restriction significantly reduced the plasma levels of total cholesterol and triglyceride, 8 weeks of exercise training had no effect on plasma total cholesterol and triglyceride levels in KKAy mice despite the improvement in glycemic control. This result was consistent with some previous studies where no significant improvement was observed in the levels of blood total cholesterol and triglyceride in obese or diabetic animal models treated with chronic exercise (Tang \& Reed 2001, Chiu et al. 2004). These findings suggest that exercise training has less of an effect than diet restriction in reducing hyperlipidemia.

In the present study, we examined levels of mRNA expression in white adipose tissue and plasma concentrations of adiponectin. It has been reported that plasma concentrations of adiponectin in obese and/or type 2 diabetic subjects were significantly lower than those in normal subjects (Hu et al. 1996, Arita et al. 1999, Hotta et al. 2000, Yang et al. 2001), and that exogenous adiponectin administration improved insulin sensitivity in obese diabetic animals (Yamauchi et al. 2001). Since increased body weight or fat cell size is highly associated with insulin resistance and the development of diabetes, decreased body weight or fat cell size might result in improvement 


\section{Skeletal muscle}

AdipoR 1

AdipoR2

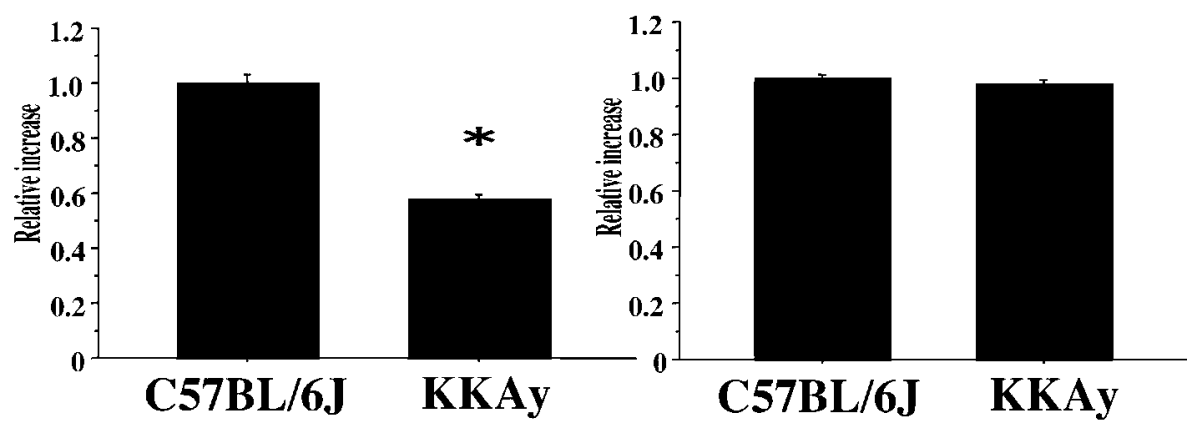

\section{Liver}

AdipoR 1

AdipoR2 *
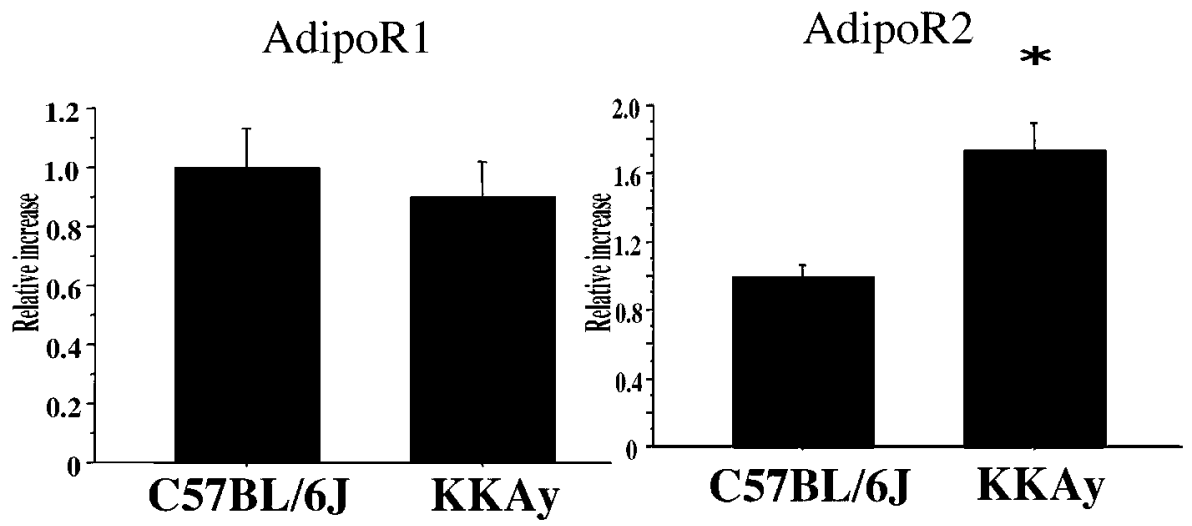

\section{White adipose tissue}
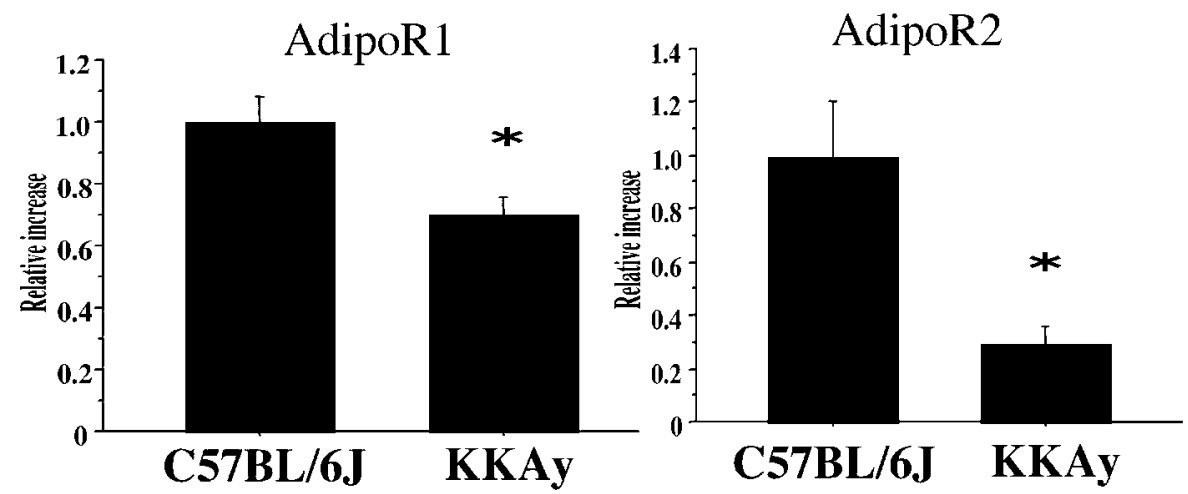

Figure 3 Expression of AdipoR1 and AdipoR2 mRNA in skeletal muscle, liver and white adipose tissue in C57BL/6 and KKAy mice. AdipoR1 and AdipoR2 mRNA expression levels were analyzed by Northern blot analysis in the skeletal muscle, liver and white adipose tissue. Results are expressed as relative activity in KKAy compared with $\mathrm{C} 57 \mathrm{BL} / 6$ mice. Each bar represents the mean \pm S.D. ${ }^{*} P<0 \cdot 05$ compared with the control group. 


\section{Skeletal muscle}

\section{AdipoR 1}

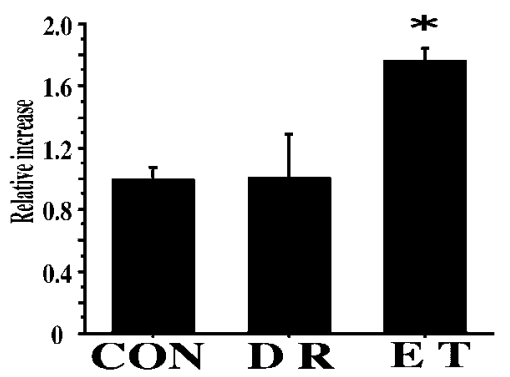

AdipoR2

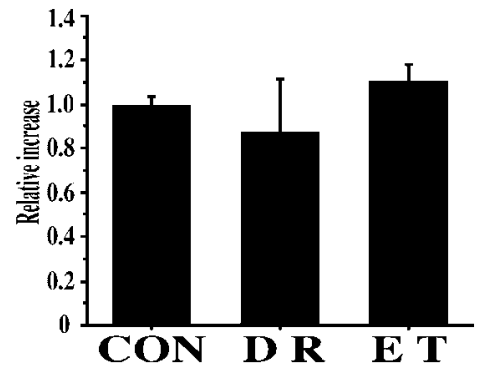

\section{Liver}

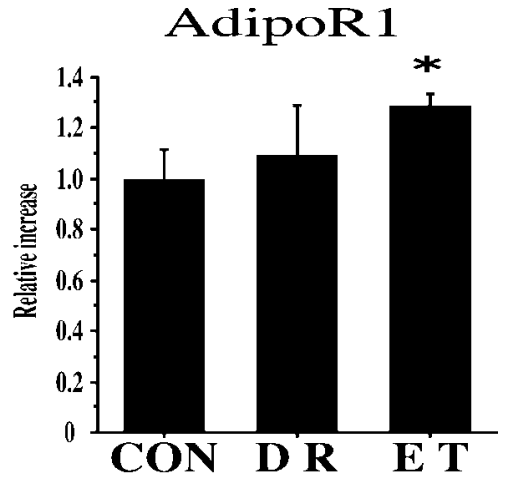

AdipoR2

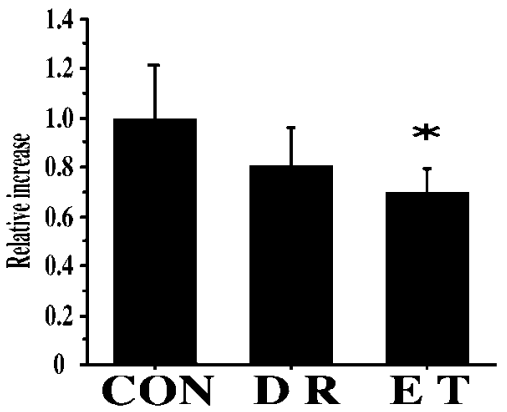

\section{White adipose tissue}

\section{AdipoR 1}

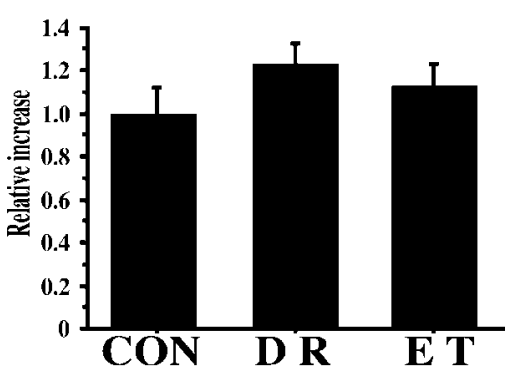

AdipoR2

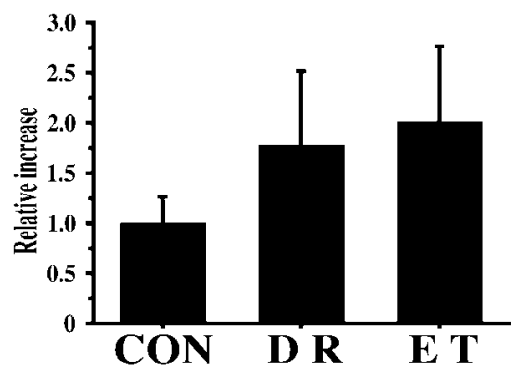

Figure 4 Expression of AdipoR1 and AdipoR2 mRNA in skeletal muscle, liver and white adipose tissue following the 8-week experimental protocol. AdipoR1 and AdipoR2 mRNA expression levels were analyzed by Northern blot analysis in the skeletal muscle, liver and white adipose tissue. Results are expressed as relative activity in the dietary restriction group (DR) and exercise training group (ET) compared with the control group (CON). Each bar represents the mean \pm S.D. ${ }^{*} P<0.05$ compared with the control group. 


\section{(A)}

\section{Skeletal muscle}

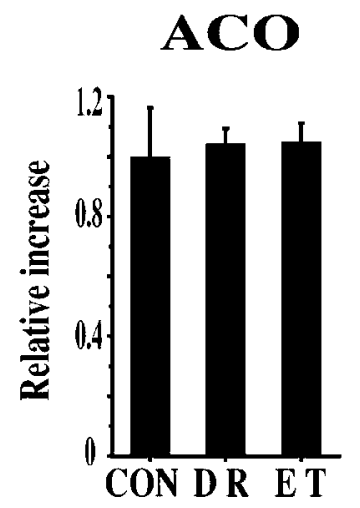

CPT1

$\operatorname{PPAR} \alpha$

$\operatorname{PPAR} \delta$
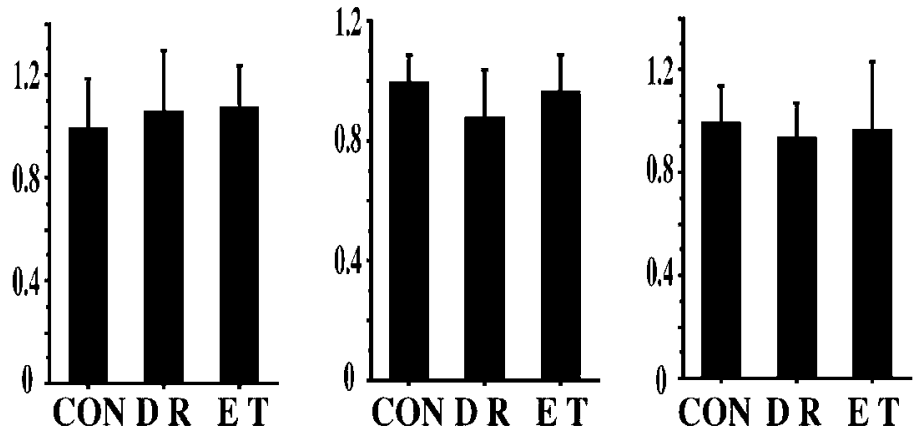

(B)
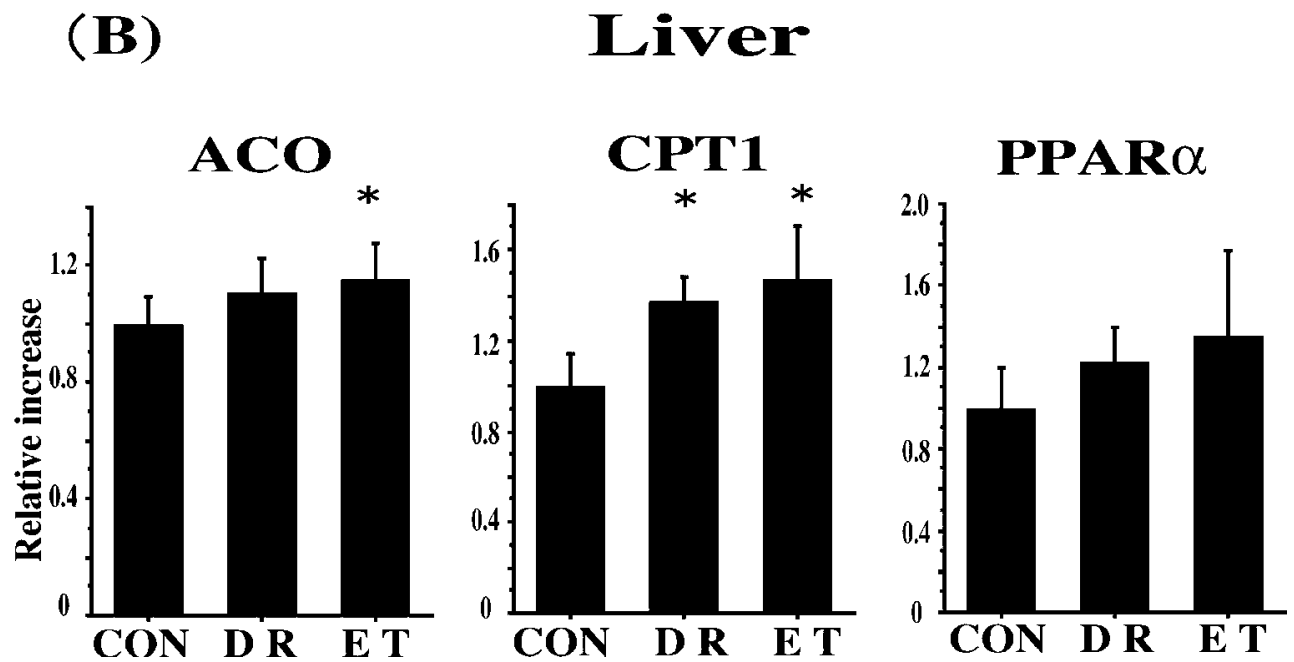

Figure $\mathbf{5}$ The expression levels of the genes related to the adiponectin receptor. The expression of ACO, CPT1, PPAR $\alpha$, and PPAR $\delta$ mRNA in skeletal muscle (A), and of ACO, CPT1, PPAR $\alpha$ mRNA in liver (B) was analyzed by Northern blot analysis. Results are expressed as relative activity in the dietary restriction group (DR) and exercise training group (ET) compared with the control group (CON). Each bar represents the mean \pm S.D. ${ }^{*} P<0 \cdot 05$ compared with the control group. ACO, acyl coenzyme A oxidase; CPT1, carnitine palmitoyl transferase 1; PPAR $\alpha$, peroxisome proliferator-activated receptor alpha; PPAR $\delta$, peroxisome proliferator-activated receptor delta.

in insulin resistance followed by increased expression and plasma concentrations of adiponectin. However, both the adiponectin gene expression and its plasma concentration showed no differences between exercise-trained mice and the control mice although the fat cell size was significantly lower in the ET group compared with the control group (Fig. 2). Similarly, in the DR group, decreased body weight and fat cell size had no effect on adiponectin expression. The finding that no change was observed in plasma adiponectin levels following exercise training is compatible with previous reports (Hulver et al. 2002,
Boudou et al. 2003, Kraemer et al. 2003, Chiu et al. 2004). Moreover, like our results, a recent report also shows that adiponectin levels are negatively associated with fat cell size in the rodent model (Florant et al. 2004). These findings suggest that exercise training had no effect on adiponectin production regardless of whether fat cell size was decreasing or not. It is possible that the decrease in fat cell size observed in our study might not have been sufficient to change the level of adiponectin expression.

Recently, adiponectin 1 and 2 receptors (AdipoR1, AdipoR2) were cloned, and it has been elucidated that 
adiponectin is bound to these receptors and mediates glucose uptake and FFA oxidation (Yamauchi et al. 2003). In rodents, AdipoR1 was ubiquitously expressed and was most abundant in skeletal muscle, whereas AdipoR2 was most abundantly expressed in the liver. We hypothesized that the expression of adiponectin receptors was regulated in target organs of insulin such as skeletal muscle and liver by exercise training, and this might promote the action of adiponectin to improve insulin sensitivity.

In comparing KKAy with lean mice, our results are in agreement with those of Tsuchida et al. (2004) indicating that the rodent model of obesity-linked insulin resistance exhibited lower levels of AdipoR1 in skeletal muscle. As expected, the expression of AdipoR1 mRNA in skeletal muscle was significantly up-regulated in the ET group. Recently, a correlation has been reported between the expression level of adiponectin receptors in skeletal muscle and insulin sensitivity in non-diabetic Mexican Americans with or without a family history of type 2 diabetes (Civitarese et al. 2004). Results from that study showed that the subjects with a family history of type 2 diabetes exhibited a lower expression level of the adiponectin receptor as compared with those subjects with no family history, and showed that the expression levels of receptors correlated positively with individual insulin sensitivity. In addition, some recent work reported that the expression levels of AdipoR1, but not AdipoR2, in skeletal muscle cells correlated with in vivo parameters of glucose metabolism, lipid metabolism and insulin secretion (Debard et al. 2004, Staiger et al. 2004). These findings suggest that the expression levels of AdipoR, especially of AdipoR1, are directly related to glucose or lipid metabolism and to insulin sensitivity in skeletal muscle. Recently, it has been suggested that insulin or hyperglycemia downregulates the expression of adiponectin receptor mRNA (Tsuchida et al. 2004, Inukai et al. 2005); therefore, it is possible that the increase in AdipoR1 observed in the present study was due to the reduction in blood insulin or glucose levels mediated by exercise training. However, in the DR group, no significant changes were observed in the expression of adiponectin receptor mRNA despite the reduction in blood insulin and glucose levels. Therefore, exercise training might directly regulate the expression of AdipoR1, thereby being involved in improvements to the diabetic state and insulin sensitivity in KKAy mice.

In contrast to the results in $\mathrm{Ob} / \mathrm{Ob}$ mice (Tsuchida et al. 2004), the expression levels of AdipoR2 mRNA unexpectedly increased in the liver of KKAy mice (Fig. 3), and such increases were recovered by exercise training (Fig. 4). The influence of increases in AdipoR2 mRNA in the liver is unclear. As AdipoR1 and AdipoR2 may form both homo- and hetero-multimers (Kadowaki \& Yamauchi 2005), the increase in AdipoR1 expression in the liver in the ET group might compensate for the decreased expression of AdipoR2.
In the present study, the expression of AdipoR1 mRNA was significantly up-regulated in both skeletal muscle and liver in the ET group. In contrast to these results, Punyadeera et al. (2005) showed that acute exercise training failed to change the expression levels of either AdipoR1 or AdipoR2 in the skeletal muscles of healthy volunteers. We speculate that the divergent results of our study arose from differences in exercise duration and intensity. Sustained exercise of sufficient intensity might provide a way to increase AdipoR 1 expression in skeletal muscle and thereby ameliorate reduced insulin sensitivity.

Adiponectin is bound to AdipoR and mediates glucose uptake and FFA oxidation through up-regulation of PPAR $\alpha$ ligand activities thereby up-regulating the expression of relative genes involved in fatty-acid oxidation, such as ACO (Yamauchi et al. 2003). To assess whether the activities of PPAR $\alpha$ were induced following the up-regulation of AdipoR1, we examined the mRNA expression levels of ACO and CPT1, the target genes for PPAR $\alpha$ transcription, and that of PPAR $\alpha$. We also examined the mRNA expression levels of PPAR $\delta$ in skeletal muscles. PPAR $\delta$ (but not PPAR $\alpha$ ) plays an important role in FFA oxidation in skeletal muscle, thus increasing the expression of genes involved in FFA oxidation (Dressel et al. 2003, Holst et al. 2003, Luquet et al. 2003). As a result, no significant change was observed in the expression levels of all genes in skeletal muscle despite the increase in AdipoR1 expression. AdipoR1 has also been reported to exert its effects through an alternative pathway in which activation of AMP-kinase is involved (Yamauchi et al. 2003, Kadowaki \& Yamauchi 2005). It is possible that the increase in AdipoR 1 induced by exercise may mediate the improvement in insulin sensitivity through such a pathway. On the other hand, the expression of ACO and CPT1 in the liver was up-regulated by exercise training. Further studies are required to elucidate whether the up-regulation of AdipoR1 induces the activation of PPAR $\alpha$, thereby increasing the expression of ACO and CPT1 in the liver.

In conclusion, chronic exercise training improves insulin sensitivity and up-regulates mRNA expression of AdipoR1 in both skeletal muscle and liver in KKAy mice. This increase in AdipoR1 expression might play a beneficial role in the improvement in insulin sensitivity through the PPAR $\alpha$-independent and -dependent pathways in muscle and liver respectively. Furthermore, AdipoR2 mRNA expression in the liver was decreased by exercise training. The influence of such a reduction of AdipoR2 expression should be studied in the near future.

\section{Funding}

The authors declare that there is no conflict of interest that would prejudice the impartiality of this scientific work. 


\section{References}

Arita Y, Kihara S, Ouchi N, Takahashi M, Maeda K, Miyagawa J, Hotta K, Shimomura I, Nakamura T, Miyaoka K, et al. 1999 Paradoxical decrease of an adipose-specific protein, adiponectin, in obesity. Biochemical and Biophysical Research Communications 257 79-83.

Boudou P, Sobngwi E, Mauvais-Jarvis F, Vexiau P \& Gautier JF 2003 Absence of exercise-induced variations in adiponectin levels despite decreased abdominal adiposity and improved insulin sensitivity in type 2 diabetic men. European Journal of Endocrinology 149 421-424.

Chiu S, Fisler JS, Espinal GM, Havel PJ, Stern JS \& Warden CH 2004 The yellow agouti mutation alters some but not all responses to diet and exercise. Obesity Research 12 1243-1255.

Civitarese AE, Jenkinson CP, Richardson D, Bajaj M, Cusi K, Kashyap S, Berria R, Belfort R, DeFronzo RA, Mandarino LJ et al. 2004 Adiponectin receptors gene expression and insulin sensitivity in non-diabetic Mexican Americans with or without a family history of type 2 diabetes. Diabetologia 47 816-820.

Debard C, Laville M, Berbe V, Loizon E, Guillet C, MorioLiondore B, Boirie Y \& Vidal H 2004 Expression of key genes of fatty acid oxidation, including adiponectin receptors, in skeletal muscle of type 2 diabetic patients. Diabetologia 47 917-925.

Dressel U, Allen TL, Pippal JB, Rohde PR, Lau P \& Muscat GE 2003 The peroxisome proliferator-activated receptor beta/delta agonist, GW501516, regulates the expression of genes involved in lipid catabolism and energy uncoupling in skeletal muscle cells. Molecular Endocrinology 17 2477-2493.

Florant GL, Porst H, Peiffer A, Hudachek SF, Pittman C, Summers SA, Rajala MW \& Scherer PE 2004 Fat-cell mass, serum leptin and adiponectin changes during weight gain and loss in yellow-bellied marmots (Marmota flaviventris). Journal of Comprehensive Physiology [B] $174633-639$.

Friedman JE, Sherman WM, Reed MJ, Elton CW \& Dohm GL 1990 Exercise training increases glucose transporter protein GLUT-4 in skeletal muscle of obese Zucker (fa/fa) rats. FEBS Letters $\mathbf{2 6 8}$ 13-16.

Holst D, Luquet S, Nogueira V, Kristiansen K, Leverve X \& Grimaldi PA 2003 Nutritional regulation and role of peroxisome proliferator-activated receptor delta in fatty acid catabolism in skeletal muscle. Biochimica et Biophysica Acta 1633 43-50.

Hotamisligil GS, Shargill NS \& Spiegelman BM 1993 Adipose expression of tumor necrosis factor-alpha: direct role in obesity-linked insulin resistance. Science 259 87-91.

Hotta K, Funahashi T, Arita Y, Takahashi M, Matsuda M, Okamoto Y, Iwahashi H, Kuriyama H, Ouchi N, Maeda K et al. 2000 Plasma concentrations of a novel, adipose-specific protein, adiponectin, in type 2 diabetic patients. Arteriosclerotic and Thrombotic Vascular Biology 20 1595-1599.

Hotta K, Funahashi T, Bodkin NL, Ortmeyer HK, Arita Y, Hansen BC \& Matsuzawa Y 2001 Circulating concentrations of the adipocyte protein adiponectin are decreased in parallel with reduced insulin sensitivity during the progression to type 2 diabetes in rhesus monkeys. Diabetes $\mathbf{5 0}$ 1126-1133.

Hu E, Liang P \& Spiegelman BM 1996 AdipoQ is a novel adipose-specific gene dysregulated in obesity. Journal of Biological Chemistry 271 10697-10703.

Hulver MW, Zheng D, Tanner CJ, Houmard JA, Kraus WE, Slentz CA, Sinha MK, Pories WJ, MacDonald KG \& Dohm GL 2002 Adiponectin is not altered with exercise training despite enhanced insulin action. American Journal of Physiology, Endocrinology and Metabolism 283 E861-E865.

Inukai K, Nakashima Y, Watanabe M, Takata N, Sawa T, Kurihara S, Awata T \& Katayama S 2005 Regulation of adiponectin receptor gene expression in diabetic mice. American Journal of Physiology, Endocrinology and Metabolism 288 E876-E882.

Kadowaki T \& Yamauchi T 2005 Adiponectin and adiponectin receptors. Endocrine Reviews 26 439-451.
Kern M, Dolan PL, Mazzeo RS, Wells JA \& Dohm GL 1992 Effect of aging and exercise on GLUT-4 glucose transporters in muscle. American Journal of Physiology 263 E362-E367.

Kraemer RR, Aboudehen KS, Carruth AK, Durand RT, Acevedo EO, Hebert EP, Johnson LG \& Castracane VD 2003 Adiponectin responses to continuous and progressively intense intermittent exercise. Medical Science and Sports Medicine 35 1320-1325.

Lonnqvist F, Arner P, Nordfors L \& Schalling M 1995 Overexpression of the obese (ob) gene in adipose tissue of human obese subjects. Nature Medicine 1950-953.

Luquet S, Lopez-Soriano J, Holst D, Fredenrich A, Melki J, Rassoulzadegan M \& Grimaldi PA 2003 Peroxisome proliferatoractivated receptor delta controls muscle development and oxidative capability. FASEB Journal 17 2299-2301. Epub 2003 Oct 2292.

Maeda K, Okubo K, Shimomura I, Funahashi T, Matsuzawa Y \& Matsubara K 1996 cDNA cloning and expression of a novel adipose specific collagen-like factor, apM1 (AdiPose Most abundant Gene transcript 1). Biochemical and Biophysical Research Communications 221 286-289.

Matthews DR, Hosker JP, Rudenski AS, Naylor BA, Treacher DF \& Turner RC 1985 Homeostasis model assessment: insulin resistance and beta-cell function from fasting plasma glucose and insulin concentrations in man. Diabetologia 28 412-419.

Punyadeera C, Zorenc AH, Koopman R, McAinch AJ, Smit E, Manders R, Keizer HA, Cameron-Smith D \& van Loon LJ 2005 The effects of exercise and adipose tissue lipolysis on plasma adiponectin concentration and adiponectin receptor expression in human skeletal muscle. European Journal of Endocrinology 152 427-436.

Scherer PE, Williams S, Fogliano M, Baldini G \& Lodish HF 1995 A novel serum protein similar to $\mathrm{C} 1 \mathrm{q}$, produced exclusively in adipocytes. Journal of Biological Chemistry 270 26746-26749.

Shimomura I, Funahashi T, Takahashi M, Maeda K, Kotani K, Nakamura T, Yamashita S, Miura M, Fukuda Y, Takemura K et al. 1996 Enhanced expression of PAI-1 in visceral fat: possible contributor to vascular disease in obesity. Nature Medicine 2 800-803.

Staiger H, Kaltenbach S, Staiger K, Stefan N, Fritsche A, Guirguis A, Peterfi C, Weisser M, Machicao F, Stumvoll M et al. 2004 Expression of adiponectin receptor mRNA in human skeletal muscle cells is related to in vivo parameters of glucose and lipid metabolism. Diabetes 53 2195-2201.

Steppan CM, Bailey ST, Bhat S, Brown EJ, Banerjee RR, Wright CM, Patel HR, Ahima RS \& Lazar MA 2001 The hormone resistin links obesity to diabetes. Nature 409 307-312.

Tang T \& Reed MJ 2001 Exercise adds to metformin and acarbose efficacy in db/db mice. Metabolism 50 1049-1053.

Tsuchida A, Yamauchi T, Ito Y, Hada Y, Maki T, Takekawa S, Kamon J, Kobayashi M, Suzuki R, Hara K et al. 2004 Insulin/Foxo1 pathway regulates expression levels of adiponectin receptors and adiponectin sensitivity. Journal of Biological Chemistry 279 30817-30822.

Weyer C, Funahashi T, Tanaka S, Hotta K, Matsuzawa Y, Pratley RE \& Tataranni PA 2001 Hypoadiponectinemia in obesity and type 2 diabetes: close association with insulin resistance and hyperinsulinemia. Journal of Clinical Endocrinology and Metabolism 86 1930-1935.

Winder WW \& Hardie DG 1996 Inactivation of acetyl-CoA carboxylase and activation of AMP-activated protein kinase in muscle during exercise. American Journal of Physiology 270 E299-E304.

Yamauchi T, Kamon J, Waki H, Terauchi Y, Kubota N, Hara K, Mori Y, Ide T, Murakami K, Tsuboyama-Kasaoka N et al. 2001 The fat-derived hormone adiponectin reverses insulin resistance associated with both lipoatrophy and obesity. Nature Medicine 7 941-946. 
Yamauchi T, Kamon J, Ito Y, Tsuchida A, Yokomizo T, Kita S, Sugiyama T, Miyagishi M, Hara K, Tsunoda M et al. 2003 Cloning of adiponectin receptors that mediate anti-diabetic metabolic effects. Nature 423 762-769.

Yang WS, Lee WJ, Funahashi T, Tanaka S, Matsuzawa Y, Chao CL, Chen CL, Tai TY \& Chuang LM 2001 Weight reduction increases plasma levels of an adipose-derived anti-inflammatory protein, adiponectin. Journal of Clinical Endocrinology and Metabolism 86 $3815-3819$
Received in final form 26 February 2006

Accepted 6 March 2006

Made available online as an

Accepted Preprint 22 March 2006 